\title{
Intermittent origin of the large violations of the fluctuation dissipation relations in an aging polymer glass
}

\author{
L. Buisson, S. Ciliberto, A. Garcimartín* \\ Ecole Normale Supérieure de Lyon, Laboratoire de Physique, \\ C.N.R.S. UMR5672, \\ 46, Allée d'Italie, 69364 Lyon Cedex 07, France
}

February 9, 2018

\begin{abstract}
The fluctuation-dissipation relation (FDR) is measured on the dielectric properties of a polymer glass (polycarbonate)in the range $20 \mathrm{mHz}-100 \mathrm{~Hz}$. It is found that after a quench below the glass transition temperature the fluctuation dissipation theorem is strongly violated. The amplitude and the persistence time of this violation are decreasing functions of frequency. At frequencies larger than $1 \mathrm{~Hz}$ it persists for about $3 h$. The origin of this violation is a highly intermittent dynamics characterized by large fluctuations. The relevance of these results for recent models of aging dynamics are discussed.
\end{abstract}

PACS:75.10.Nr, 77.22.Gm, 64.70.Pf, 05.20.-y

When glassy materials are quenched from above their glass transition temperature $T_{g}$ to a temperature lower than $T_{g}$, any response function of

*Permanent address: Departamento de Física, Facultad de Ciencias, Universidad de Navarra, E-31080 Pamplona, Spain. 
these materials depends on the time $t_{w}$ elapsed from the quench: they are aging [1]. A widely studied question, is how the temperature of these systems can be defined. Recent theories [2] based on the description of spin glasses by a mean field approach propose to extend the concept of temperature using a Fluctuation Dissipation Relation (FDR) which generalizes for a weakly out of equilibrium system the Fluctuation Dissipation Theorem (FDT) . (For a review see ref. [3, 4]). At equilibrium, FDT relates the fluctuation spectral density of a variable $\mathrm{V}$ to the response $\chi_{V q}(f)$ of $\mathrm{V}$ to a perturbation of its conjugated variable $\mathrm{q}$ at frequency f:

$$
S(f)=\frac{2 K_{B} T}{\pi f} \operatorname{Im}\left[\chi_{V q}(f)\right]
$$

where $S(f)=<|V(f)|^{2}>$ is the fluctuation spectral density of $V, K_{B}$ is the Boltzmann constant, T the temperature of the system and $\operatorname{Im}\left[\chi_{V q}(f)\right]$ is the imaginary part of $\chi_{V q}(f)$. When the system is not in equilibrium FDT, that is eq1 may fail. Because of the slow dependence on $t_{w}$ of the response functions, it has been proposed to use an FDR which generalizes eq 1 and which can be used to define an effective temperature $T_{\text {eff }}\left(f, t_{w}\right)$ of the system 4]:

$$
T_{e f f}\left(f, t_{w}\right)=\frac{S\left(f, t_{w}\right) \pi f}{\operatorname{Im}\left[\chi_{V q}\left(f, t_{w}\right)\right] 2 K_{B}}
$$

It is clear that if eq1 is satisfied $T_{\text {eff }}=T$, otherwise $T_{\text {eff }}$ turns out to be a decreasing function of $t_{w}$ and $f$. The physical meaning of eq 2 is that there is a time scale (for example $t_{w}$ ) which allows to separate the fast processes from the slow ones. In other words the low frequency modes, such that $f t_{w}<1$, relax towards equilibrium much slower than the high frequency ones which rapidly relax to the temperature of the thermal bath. This striking behavior has been observed in several numerical models of aging [5]-[10], which show that eq 2 is a good definition of temperature in the thermodynamic sense [4]. The experimental analysis of the dependence of $T_{\text {eff }}\left(f, t_{w}\right)$ on $f$ and $t_{w}$ is very useful to distinguish among different models of aging because the FDT violations are model dependent ([4]-[10]).

Recently, a few experiments have analyzed this problems in real materials [1], [12, 13]. The violation of FDT measured in an experiment on a spin glass [13] seems to be in agreement with theoretical predictions. The experiment on the dielectric measurements on glycerol [11] is a single frequency experiment. Thus cannot give insight on the time evolution of the spectrum. 

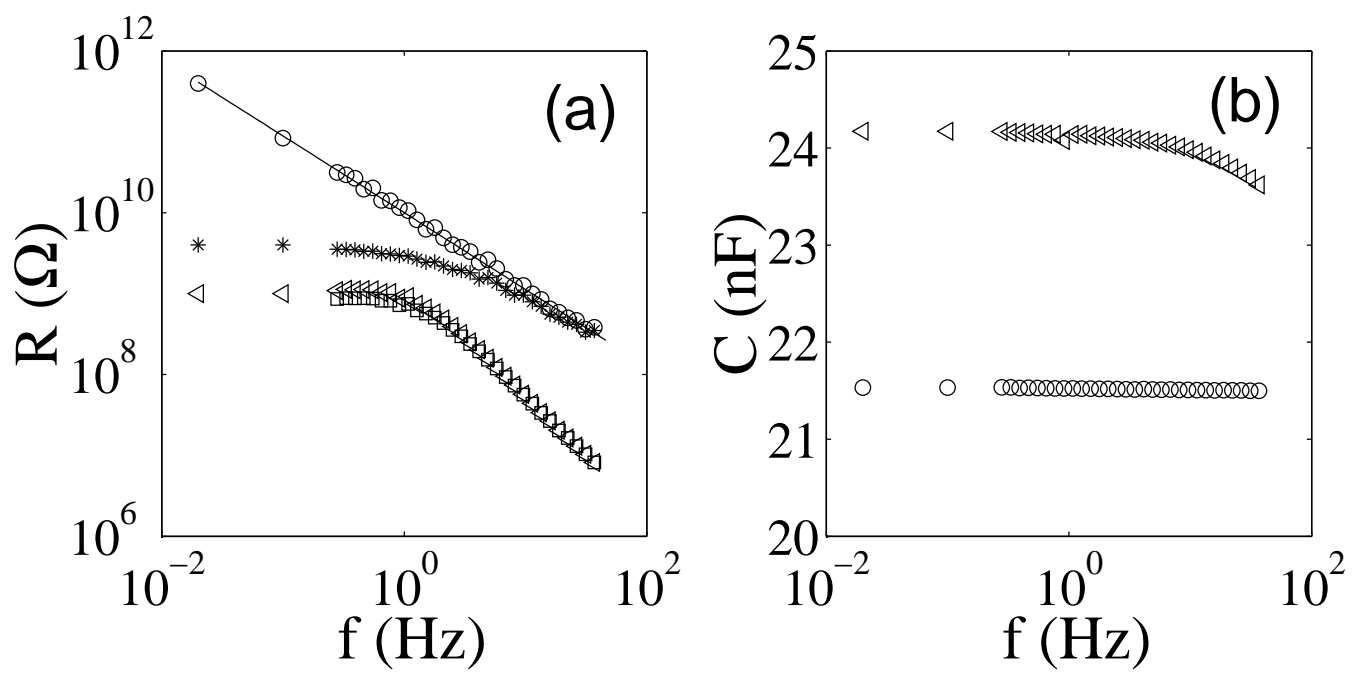

Figure 1: (a) Polycarbonate resistance $R$ as a function of frequency measured at $T_{i}=433 K(\triangleleft)$ and at $T_{f}=333 K(\circ)$. The effect of the $4 G \Omega$ input resistance is also shown at $T=433 K(\square)$ and at $T=333 K(*)$. b) Polycarbonate capacitance versus frequency measured at $T_{i}=433 K(\triangleleft)$ and at $T_{f}=333 k(\circ)$.

The experiment on colloidal glasses 12 presents only a qualitative agreement with theory. Specifically it shows that the persistence time of the violation is very long and the amplitude of the violation is huge.

In order to give more insight into this problem we have done wide band $(20 \mathrm{mHz}, 100 \mathrm{~Hz})$ measurements of the dielectric susceptibility and of the polarization noise in a polymer glass. In this letter we present results which show a strong violation of the FDT in a polymer glass quenched from the molten state to below its glass-transition temperature. $T_{\text {eff }}$ defined by eq2 slowly relaxes towards the bath temperature. The violation is observed even at $f t_{w}>>1$ and it may last for more than $3 h$ for $f>1 H z$. The polymer used is Makrofol DE 1-1 C, a bisphenol A polycarbonate, with $T_{g} \simeq 419 K$, produced by Bayer in form of foils. This material has been chosen because it has a wide temperature range of strong aging [1] and is totally amorphous. Many studies of the dielectric susceptibility of this material exist, but no one had an interest on the problem of noise measurements. In our experiment polycarbonate is used as the dielectric of a capacitor. The capacitor is composed by 14 cylindrical capacitors in parallel in order to reduce the resistance 
of the sample and to increase its capacity. Each capacitor is made of two aluminum electrodes, $12 \mu \mathrm{m}$ thick, and by a disk of polycarbonate of diameter $12 \mathrm{~cm}$ and thickness $125 \mu \mathrm{m}$. The 14 capacitors are sandwiched together and put inside two thick aluminum plates which contain an air circulation used to regulate the sample temperature within a few percent. This mechanical design of the capacitor is very stable and gives very reproducible (better than $1 \%$ ) results even after many temperature quenches. The capacitor is inside two Faraday screens to insulate it from external noise. Fast quench of about $50 \mathrm{~K} / \mathrm{min}$ are obtained by injecting Nitrogen vapor in the air circulation of the aluminum plates. The electrical impedance of the capacitor is $Z\left(f, t_{w}\right)=R /(1+i 2 \pi f R C)$, where $C$ is the capacitance and $R$ is a parallel resistance which accounts for the complex dielectric susceptibility. It is measured using a Novocontrol Dielectric Analyzer. The noise spectrum of this impedance $S_{Z}\left(f, t_{w}\right)$ is:

$$
S_{Z}\left(f, t_{w}\right)=\frac{4 K_{B} T_{e f f}\left(f, t_{w}\right) R}{1+(2 \pi f R C)^{2}}
$$

where $T_{\text {eff }}$ is the effective temperature of the sample. The polarization noise of $Z$ is sent to an amplifier whose input resistance is $R_{i}=4 G \Omega$. The output signal of the amplifier is directly acquired by a NI4462 card. The measured spectrum at the amplifier input is:

$$
\begin{aligned}
S_{V}\left(f, t_{w}\right) & =\frac{4 K_{B} R R_{i}\left(T_{e f f}\left(f, t_{w}\right) R_{i}+T_{R} R\right)}{\left(R+R_{i}\right)^{2}+\left(2 \pi f R R_{i} C\right)^{2}}+ \\
& +\frac{S_{\xi}(f) R R_{i}}{\left(R+R_{i}\right)^{2}+\left(2 \pi f R R_{i} C\right)^{2}}+S_{\eta}(f)
\end{aligned}
$$

where $T_{R}$ is the temperature of $R_{i}$ and $S_{\eta}$ and $S_{\xi}$ are respectively the voltage and the current noise spectrum of the amplifier. The desired statistical accuracy of $S_{V}\left(f, t_{w}\right)$ is reached by averaging the results of many experiments. In each of these experiments the sample is first heated to $T_{i}=433 \mathrm{~K}$. It is maintained at this temperature for 4 hours to reinitialize its thermal history. Then it is quenched from $T_{i}$ to $T_{f}=333 \mathrm{~K}$ in about 2 minutes. The origin of aging time $t_{w}$ is the instant when the capacitor temperature is at $T_{g} \simeq 419 \mathrm{~K}$, which of course may depend on the cooling rate. However adjustment of $T_{g}$ of a few degrees will shift the time axis by at most 30 s, without affecting our results.

At $T_{f}=333 \mathrm{~K}$ the main relaxation process of polycarbonate are well outside the frequency range of our measurements, specifically the $\alpha$ relaxation 
frequency is smaller than $10^{-10} \mathrm{~Hz}$ and the $\beta$ relaxation frequency is larger than $10^{6} \mathrm{~Hz}$. Thus we are testing a frequency range which is far away the two relaxation frequencies. In fig $\mathbb{1}$ (a) and (b), we plot the measured values of $R$ and $C$ as a function of $f$ at $T_{i}$ and at $T_{f}$ for $t_{w} \geqslant 200 s$. We see that lowering temperature $R$ increases and $C$ decreases. At $T_{f}$ aging is small and extremely slow. Thus for $t_{w}>200 \mathrm{~s}$ the impedance can be considered constant without affecting our results. From the data plotted in fig 1 (a) and (b) one finds that $R=10^{10}(1 \pm 0.05) f^{-0.95 \pm 0.01} \Omega$ and $C=(21.5 \pm 0.05) n F$. In fig 1 (a) we also plot the total resistance at the amplifier input which is the parallel of the capacitor impedance with $R_{i}$. We see that at $T_{f}$ the input impedance of the amplifier is negligible for $f>10 H z$, whereas it has to be taken into account at slower frequencies.

Fig 2(a) represents the evolution of $S_{V}\left(f, t_{w}\right)$ after a quench. Each spectrum is obtained as an average in a time window starting at $t_{w}$. The time window increases with $t_{w}$ so to reduce errors for large $t_{w}$. Then the results of 7 quenches have been averaged. At longest time $\left(t_{w}=1\right.$ day $)$ the equilibrium FDT prediction (continuous line) is quite well satisfied. FDT is strongly violated for all frequencies at short times. Then high frequencies relax on the FDT, but there is a persistence of the violation for lower frequencies. The amount of the violation can be estimated by the best fit of $T_{\text {eff }}\left(f, t_{w}\right)$ in eq4 where all other parameters are known. We start at very large $t_{w}$ when the system is relaxed and $T_{\text {eff }}=T$ for all frequencies. Inserting the values in eq 4 and using the $S_{V}$ measured at $t_{w}=1$ day we find $T_{\text {eff }} \simeq 333 \mathrm{~K}$, within error bars for all frequencies (see fig $2 \mathrm{~b}$ ). At short $t_{w}$ data show that $T_{\text {eff }}\left(f, t_{w}\right) \simeq T_{f}$ for $f$ larger than a cut-off frequency $f_{o}\left(t_{w}\right)$ which is a function of $t_{w}$. In contrast, for $f<f_{o}\left(t_{w}\right), T_{\text {eff }}\left(f, t_{w}\right) \propto f^{-A\left(t_{w}\right)}$, with $A\left(t_{w}\right) \simeq 1$. This frequency dependence of $T_{e f f}\left(f, t_{w}\right)$ is quite well approximated by

$$
T_{e f f}\left(f, t_{w}\right)=T_{f}\left[1+\left(\frac{f}{f_{o}\left(t_{w}\right)}\right)^{-A\left(t_{w}\right)}\right]
$$

where $A\left(t_{w}\right)$ and $f_{o}\left(t_{w}\right)$ are the fitting parameters. We find that $1<A\left(t_{w}\right)<$ 1.2 for all the data set. Furthermore for $t_{w} \geq 250 \mathrm{~s}$, it is enough to keep $A\left(t_{w}\right)=1.2$ to fit the data within error bars. For $t_{w}<250 \mathrm{~s}$ we fixed $A(t)=1$. Thus the only free parameter in eq 5 is $f_{o}\left(t_{w}\right)$. The continuous lines in fig 2(a) are the best fits of $S_{V}$ found inserting eq 5in eq, 4 In fig.2(b) we plot the estimated $T_{e f f}\left(f, t_{w}\right)$ as a function of frequency at different $t_{w}$. Just after the quench $T_{\text {eff }}\left(f, t_{w}\right)$ is much larger than $T_{f}$ in all the frequency interval. High frequencies rapidly decay towards the FDT prediction whereas 

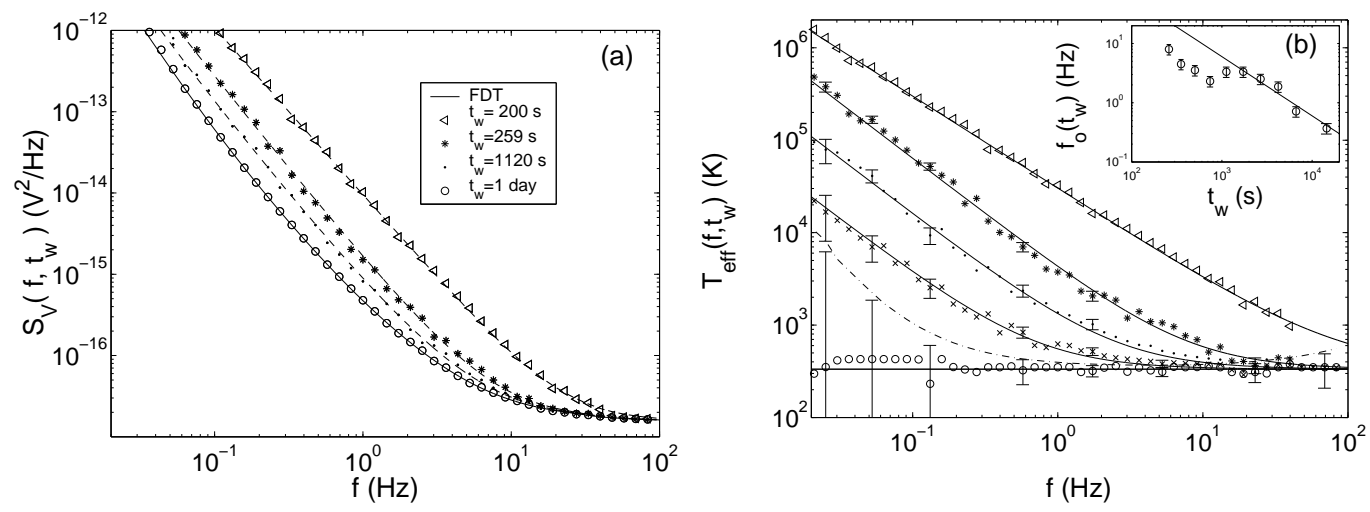

Figure 2: (a) Noise power spectral density $S_{V}\left(f, t_{w}\right)$ measured at $T_{f}=333 K$ and different $t_{w}$. The spectra are the average over seven quenches. The continuous line is the FDT prediction. Dashed lines are the fit obtained using eq4 and eq 5 (see text for details). (b) Effective temperature vs frequency at $T_{f}=333 \mathrm{~K}$ for different aging times: $(\triangleleft) t w=200 \mathrm{~s},(*) t w=260 \mathrm{~s}$, - $t w=2580 \mathrm{~s},(\times) t_{w}=6542 \mathrm{~s},(\circ) t_{w}=1$ day. The continuous lines are the fits obtained using eq 5 . The horizontal straight line is the FDT prediction. The dot dashed line corresponds to the limit where the FDT violation can be detected. In the inset the frequency $f_{o}\left(t_{w}\right)$, defined in eq 5 is plotted as a function of $t_{w}$. The continuous line is not a fit, but it corresponds to $f_{o}\left(t_{w}\right) \propto 1 / t_{w}$.

at the smallest frequencies $T_{\text {eff }} \simeq 10^{5} \mathrm{~K}$. Moreover low frequencies decay more slowly than high frequencies and the evolution of $T_{e f f}\left(f, t_{w}\right)$ towards equilibrium is very slow. From the data of Fig 2(b) and eq 5 it is easy to see that $T_{\text {eff }}\left(f, t_{w}\right)$ can be superposed onto a master curve by plotting them as a function of $f / f_{o}\left(t_{w}\right)$. The function $f_{o}\left(t_{w}\right)$ is a decreasing function of $t_{w}$, but the dependence is not a simple one, as it can be seen in the inset of fig $2(b)$. The continuous straight line is not a fit, it represents $f_{o}\left(t_{w}\right) \propto 1 . / t_{w}$ which seems a reasonable approximation for these data. For $t_{w}>10^{4} \mathrm{~s}$ we find the $f_{o}<1 H z$. Thus we cannot follow the evolution of $T_{\text {eff }}$ anymore because the contribution of the experimental noise on $S_{V}$ is too big, as it is shown in Fig (2) by the increasing of the error bars for $t_{w}=1$ day and $f<0.1 \mathrm{~Hz}$.

Before discussing these experimental results we want to compare them to the single frequency experiment performed on glycerol [11. In this experiment, $T_{\text {eff }}$ has been measured only at $7 \mathrm{~Hz}$ and nothing can be said on the 
dependence of $T_{\text {eff }}$ on frequency. This is a crucial point in order to compare the observed dynamics to that of models 44 and to see whether there is a connection between $T_{\text {eff }}$ and the concept of fictive temperature (see discussion in ref. [4]). The strong frequency dependence of $T_{\text {eff }}$ suggest that such a comparison will make sense only for $f \rightarrow 0$. Therefore it would be interesting to check whether at shorter times and at lower frequencies large $T_{\text {eff }}$ could be observed in glycerol too.

To compare with theoretical predictions [2, 4] and recent spin glass experiment [13] we may plot the integrated response $R\left(t, t_{w}\right)$ as a function of the correlation $C\left(t, t_{w}\right)$. The latter is obtained inserting the measured $T_{\text {eff }}\left(f, t_{w}\right)$ in eq 3 and Fourier transforming it. $R\left(t, t_{w}\right)$ can be computed by Fourier transforming $\operatorname{Real}\left[Z\left(f, t_{w}\right)\right]$. FDR now takes the form [4]:

$$
-C\left(t, t_{w}\right)+C\left(t_{w}, t_{w}\right)=K_{B} T_{e f f}\left(t, t_{w}\right) R\left(t, t_{w}\right)
$$

The study of the correlations functions will be the object of a longer reports 14. Here we want only to stress that the selfsimilarity of $T_{\text {eff }}$ for $t_{w}>300 s$ (see eq implies that also $C\left(t_{w}, t\right)$ can be scaled onto a single master curve by plotting $C\left(t_{w}, t\right)$ as a function $\left(t-t_{w}\right) / t_{o}\left(t_{w}\right)$, where $t_{o}\left(t_{w}\right)$ is an increasing function of $t_{w}$ : approximately $t_{o}\left(t_{w}\right) \propto 1 / f_{o}\left(t_{w}\right)$ for $t_{w}>300 \mathrm{~s}$. The self-similarity of correlation functions, found on our dielectric data, is a characteristic of the universal picture of aging [3, 4, 15, 15], which has been also observed in spin-glass experiment [13] and in the structure function of the dynamic light scattering of colloidal gels [16]. Thus our results show that this picture of aging applies also to the dielectric noise of a polymer. Furthermore the study of $R\left(t, t_{w}\right)$ as a function $\left(-C\left(t, t_{w}\right)+C\left(t_{w}, t_{w}\right)\right) / K_{B}$ confirms the spectral analysis of fig 2, that is $T_{\text {eff }}$ is huge for long $\left(t-t_{w}\right)$. This result is quite different to what has been observed in recent experiments on spin glasses where $T_{\text {eff }} \simeq 5 T_{g}$ has been measured [13.

A large $T_{\text {eff }}$ is not specific to our system but it has been observed in domain growth models [4, 8, and in models controlled by activation processes [3. 17, 18, 19]. The question is whether these models may have some connections with our observations. In order to understand the origin of such large deviations in our experiment we have analyzed the noise signal. We find that the signal is characterized by large intermittent events which produce low frequency spectra proportional to $f^{-\alpha}$ with $\alpha \simeq 2$. Two typical signals recorded at $1500 \mathrm{~s}<t_{w}<1900 \mathrm{~s}$ and $t_{w}>75000 \mathrm{~s}$ are plotted in fig 3 . We clearly see that in the signal recorded at $1500 s<t_{w}<1900 s$ there are 
very large bursts which are on the origin of the frequency spectra previously. In contrast in the signal (fig $3 \mathrm{~b}$ ), which was recorded at $t_{w}>75000 \mathrm{~s}$ when FDT is not violated, the bursts are totally disappeared. It is interesting to notice that this kind of dynamics could be a common feature of several aging systems (see also ref.[20]). Indeed intermittency has been also observed in dielectric local measurements of polymers 21] and in the diffusing wave spectroscopy measurements in gels 22 .
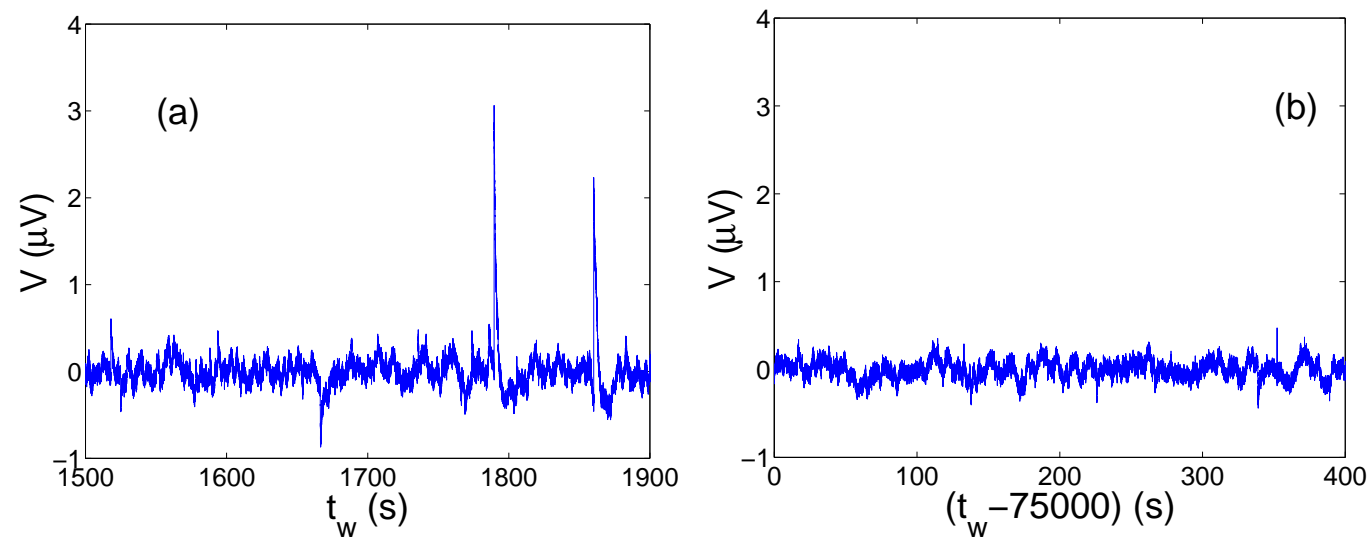

Figure 3: Voltage noise signal in polycarbonate Typical noise signal of polycarbonate measured at $1500 s<t_{w}<1900 s$ (a) and $t_{w}>75000 s$ (b)
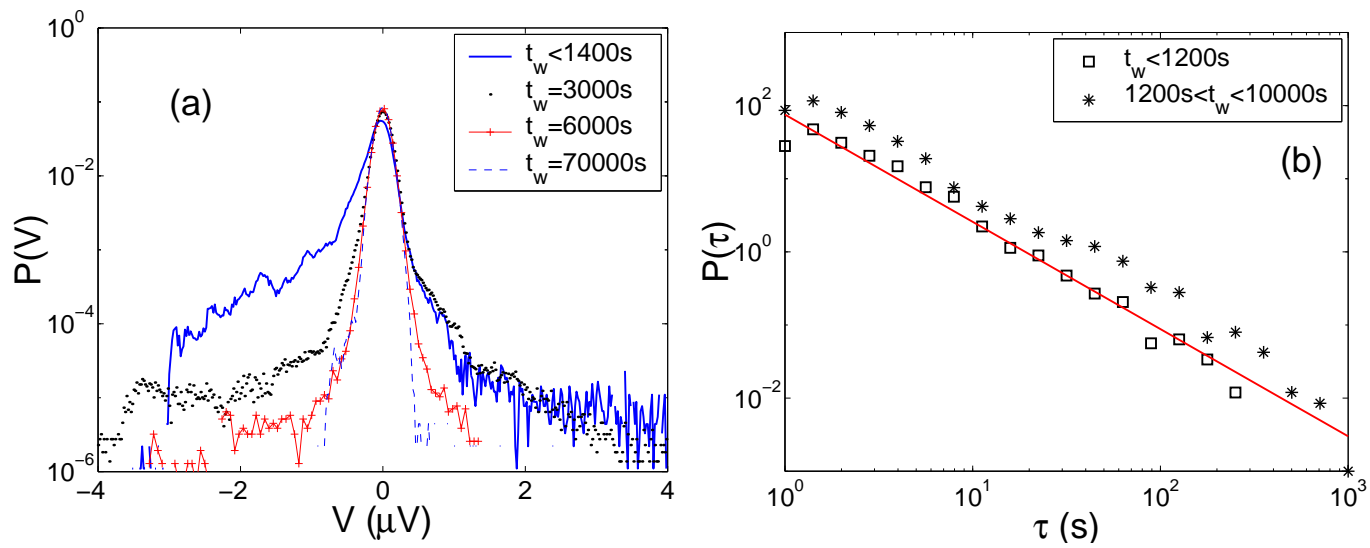

Figure 4: PDF of voltage noise in polycarbonate Typical PDF of the noise signal of polycarbonate measured at various $t_{w}$ 
To further characterize the observed dynamics we have computed the probability density function (PDF) of the signals, which is plotted in fig $⿴ 囗 4$ (a) for different $t_{w}$. We clearly see that the PDF, measured at small $t_{w}$, has very high tails which become smaller and smaller at large $t_{w}$. Finally the Gaussian profiles is recovered after 24h. In a more quantitative way we find that the Kurtosis $K u$ (which is 0 for a Gaussian) of the distribution is a decreasing function of time which can be roughly approximated by: $K u=(6 \pm 1)\left(t_{w} / 1000\right)^{-0.55 \pm 0.05)}$. The time interval $\tau$ between two successive pulses is power law distributed. In order to study the distribution $P\left(\tau, t_{w}\right)$ of $\tau$ we have first selected the signal fluctuations with amplitude larger than a fixed threshold, which has been chosen between 3 and 4 standard deviations of the equilibrium noise,i.e. the noise predicted by FDT. We have then measured the time intervals $\tau$ between two successive large fluctuations. The PDF $P\left(\tau, t_{w}\right)$ computed for $t_{w}<20 \mathrm{~min}$ and for 20min $<t_{w}<3 h$ is plotted in fig प $\mathrm{b})$. We clearly see that $P\left(\tau, t_{w}\right)$ is a power law, specifically $P(\tau) \propto \frac{1}{\tau^{1+\mu}}$ with $\mu \simeq 0.4 \pm 0.1$. This result agrees with one of the predictions of the trap model, [17, which presents non trivial violation of FDT associated to an intermittent dynamics. In the trap model $\tau$ is a power law distributed quantity with an exponent $1+\mu$ that, in the glass phase, is smaller than 2 . However there are important differences between the dynamics of our system and that of the trap model. Indeed in this model one finds short and large $\tau$ for any $t_{w}$ which is in contrast with our system because the probability of finding short $\tau$ seems to decrease as a function of $t_{w}$. But this effect could be a consequence of the imposed threshold. It seems that there is no correlation between the $\tau$ and the amplitude of the associated bursts. Finally the maximum distance $\tau_{\max }$ between two successive pulses grows as function $t_{w}$ logarithmically, that is $\tau_{\max }=\left[10+152 \log \left(t_{w} / 300\right)\right] s$ for $t_{w}>300 s$. This slow relaxation of the number of events per unit time shows that the intermittency is related to aging.

In conclusion, we have observed a large violation of FDT in the dielectric thermal noise of an aging polymer glass. Such a large violation is produced by rare events of high amplitude. The important question is now to understand the physical origin of these big events in the electrical thermal noise of the sample. A possibility is the triboelectricity produced by the aging induced stress relaxation in the sample. Mechanical and acoustical measurements performed in parallel with dielectric measurements could clarify this problem. Work is in progress.

We acknowledge useful discussion with and L. Bellon, L. Cugliandolo 
and J.P. Bouchaud. We thank P. Metz and F. Vittoz for technical support. This work has been partially supported by the Région Rhône-Alpes contract "Programme Thématique : Vieillissement des matériaux amorphes".

\section{References}

[1] L.C. Struik, Physical aging in amorphous polymers and other materials (Elsevier, Amsterdam, 1978).

[2] L.Cugliandolo, J.Kurchan, Phys.Rev.Lett., 71, 173, (1993).

[3] J.P. Bouchaud, L.F. Cugliandolo, J. Kurchan, M. Mézard, in Spin Glasses and Random Fields, ed A.P. Young (World Scientific, Singapore 1998).

[4] L. Cugliandolo, J. Kurchan, L. Peliti, Phys.Rev. E 55, 3898 (1997).

[5] W. Kob, J.L. Barrat,Europhys. Lett.,46 (5),pp. 637-642 (1999). W. Kob, J.L. Barrat, Phys.Rev.Lett.,78, 4581 (1997);

[6] G. Parisi, Phys.Rev.Lett.,79, 3660 (1997).

[7] L. Berthier, J.L. Barrat, J. Kurchan, Phys. Rev. E, 61, 5464 (2000).

[8] A. Barrat, Phys.Rev. E 57, 3629 (1998).

[9] M. Sellitto, Eur.Phys.J., B4, 135 (1998).

[10] E. Marinari, G. Parisi, F. Ricci-Tersenghi, J.J. Ruiz-Lorenzo, J.Phys.A: Math.Gen., 31, 2611 (1998).

[11] T.S. Grigera, N. Israeloff, Phys.Rev.Lett., 83,5038(1999).

[12] L. Bellon, S. Ciliberto, C. Laroche, Europhys. Lett., 53, 511 (2001).

[13] D. Herrisson, M. Ocio, Phys.Rev.Lett., 88, 257702(2002).

[14] L. Buisson, L. Bellon, S. Ciliberto, J. of Physics: Cond. Matt. (to be published).

[15] L. Berthier, J.P. Bouchaud cond-mat/0202069v1 
[16] L. Cipelletti, S. Manley, R.C. Ball, D.A. Weitz, Phys.Rev.Lett. 84, 2275 (2000).

[17] J.P. Bouchaud,D.S. Dean,J.Phys.I France,5, $265 \quad$ (1995). E.Bertin,J.P.Bouchaud, cond-mat/0112187

[18] S. Fielding, P. Sollich, Phys. Rev. Lett., 88, 50603-1, (2002).

[19] A. Perez-Madrid, D. Reguera, J.M. Rubi, Origin of the Violation of the Fluctuation-Dissipation Theorem in Systems with Activated Dynamics. cond-mat/0210089.

[20] L. Cugliandolo, Hetrogeneity and local fluctuations in glassy systems, preprint 2003.

[21] E. Vidal Russel, N.E. Israeloff, Nature 408,695 (2000).

[22] L. Cipelletti, et al.,J. Phys. Condens. Matter, (2003) 